\title{
Anatomic and flow dynamic considerations for safe right axillary artery cannulation
}

\author{
Julia Hillebrand, MD, ${ }^{a}$ Moritz Anton Konerding, $\mathrm{MD}, \mathrm{PhD},{ }^{\mathrm{b}}$ Mathias Koch, ${ }^{\mathrm{a}}$ Tim Kaufmann, $\mathrm{PhD},{ }^{\mathrm{c}}$ \\ Ulrich Steinseifer, $\mathrm{PhD},{ }^{\mathrm{c}}$ Anton Moritz, MD, PhD, ${ }^{\mathrm{a}}$ and Omer Dzemali, $\mathrm{MD}, \mathrm{PhD}^{\mathrm{d}}$
}

\begin{abstract}
Objectives: Neuroprotection is of paramount interest in cardiac surgery. Right axillary artery cannulation is well established in aortic surgery because it significantly improves survival and outcome, but malperfusion of the right brain after direct cannulation has been reported. Anatomically, 4 vessel segments are potentially amenable for cannulation of the subclavian and axillary arteries. Clinical studies vary widely in dissection sites and cannulation techniques. We investigated critical flow dynamics in the right brain caused by arterial inflow after direct cannulation and specified cannulation positions that provide optimal cerebral perfusion.
\end{abstract}

Methods: Distances from the lateral margin of the axillary artery and the subclavian artery to the origin of the vertebral artery were measured in 14 human corpses by a flexible ruler. We calculated the hemodynamics within the vertebral artery, depending on different positions of the cannula tip, in a computer-calculated model.

Results: The mean distance from the axillary artery to the vertebral artery was $8.5 \mathrm{~cm}$, and the mean distance from the subclavian artery to the vertebral artery was $6.7 \mathrm{~cm}$. Computed flow calculations demonstrated reversed flow in the vertebral artery when the cannula tip was positioned too close to its orifice. To ensure safe supraaortic flow, a cannula can be inserted securely up to $6.0 \mathrm{~cm}$ into the axillary artery and $4.2 \mathrm{~cm}$ into the subclavian artery.

Conclusions: Direct cannulation of the right axillary artery can lead to cerebral malperfusion, caused by an obstruction of the vertebral artery's orifice by the arterial cannula or a subclavian steal phenomenon due to flow reversal. The safety of direct axillary artery cannulation can be improved by a well-considered dissecting site and insertion length of the cannula. (J Thorac Cardiovasc Surg 2013;146:467-71)

The subclavian artery (SA) is increasingly used for arterial inflow in surgery of the ascending aorta and aortic arch because neurologic complications and mortality ${ }^{1-3}$ can be reduced. Until now, the optimal cannulation technique has been under debate. ${ }^{1}$

Further decrease of neurologic impairment has been reported by several groups using a side graft for cannulation of the SA. ${ }^{4-7}$ However, in these studies cannulation of the axillary artery (AA) and SA was performed without discriminating the results related to either cannulation site. Moreover, the types of cannulas used, insertion techniques (eg, routine use of a guidewire or not), and dissection sites varied widely.

\footnotetext{
From the Department of Thoracic and Cardiovascular Surgery, ${ }^{\text {a }}$ Johann Wolfgang Goethe-University Hospital, Frankfurt am Main, Germany; Institute of Functional and Clinical Anatomy, ${ }^{\mathrm{b}}$ University Medical Center of the Johannes Gutenberg-University, Mainz, Germany; Helmholtz-Institute for Biomedical Engineering, ${ }^{c}$ Rheinisch-Westfälische Technische Hochschule Aachen, Aachen, Germany; and Department of Cardiac Surgery, ${ }^{\mathrm{d}}$ University Heart Center Triemli, Zurich, Switzerland.

Disclosures: Authors have nothing to disclose with regard to commercial support.

Received for publication Nov 28, 2012; revisions received Jan 22, 2013; accepted for publication Feb 12, 2013

Address for reprints: Julia Hillebrand, MD, Department of Cardiac Surgery, University Hospital of the Westfälische Wilhelms-University Münster, Albert-SchweitzerCampus 1, D-48159 Muenster, Germany (E-mail: Julia.Hillebrand@ukmuenster.de). 0022-5223/\$36.00

Copyright (c) 2013 Published by Elsevier Inc. on behalf of The American Association for Thoracic Surgery

http://dx.doi.org/10.1016/j.jtcvs.2013.02.023
}

As shown in Figure 1, there are 4 anatomically defined segments potentially amenable for cannulation of the SA or AA: (1) the distal segment of the SA, which is located between the clavicle and the first rib; (2) the proximal segment of the AA, defined by the lateral margin of the first rib to the medial margin of the minor pectoralis muscle; (3) the middle segment of the AA (vessel part dorsal the minor pectoralis muscle); and (4) the distal segment of the AA (lateral margin of the minor pectoralis muscle to the lower margin of the major pectoralis muscle).

The classic surgical exposure of the distal SA and the proximal AA is achieved by an access parallel to the clavicle. ${ }^{8}$ When dissecting the distal segment of the SA just beneath the major pectoralis muscle, the tip of a directly inserted cannula may come in close proximity to the orifice of the vertebral artery (VA). Because of the flexibility of the surrounding tissue in this area, it is difficult to measure the distance to the VA exactly once the vessel is looped and tension is exerted to expose the artery. Within the segment of the VA's origin, the SA changes its course from central upward to outward and downward. This fact might aggravate local flow disturbance when the tip of the arterial cannula is positioned within this potentially critical area.

We performed the present study to analyze critical flow dynamics in the right supra-aortic vessels caused by AA inflow after direct cannulation. Our aim was the specification 


\section{Abbreviations and Acronyms \\ AA $=$ axillary artery \\ SA = subclavian artery \\ $\mathrm{VA}=$ vertebral artery \\ WSS $=$ wall shear stress}

of the optimal vascular dissection site to provide safe cerebral perfusion.

\section{MATERIAL AND METHODS \\ Anatomic Study}

Our anatomic investigations were performed in the Institute of Functional and Clinical Anatomy of the University Medical Center of the Johannes Gutenberg-University in Mainz, Germany. According to the described anatomic segmentation of the SA and AA shown in Figure 1, we measured the following distances in 14 cadavers of whole body donators ( 7 female and 7 male specimens). All donators gave their bodies with written informed consent at lifetime to the Institute of Functional and Clinical Anatomy for educational and research purposes. The cadavers were fixed by arterial perfusion through the femoral artery with $1.2 \%$ formaldehyde and alcohol and stored in an embalming facility.

Surgical access was imitated by subclavicular incision. After dissection of the skin and the overlying structures, the vessels were measured with a flexible ruler in situ unstretched because we used fixed corpses with limited tissue elasticity. The measurement protocol comprised the distances between the VA's origin to the end of the SA and the end of the distal segment of the right AA.

\section{Computer Model for Hemodynamic Calculations}

The Helmholtz-Institute for Biomedical Engineering of the RheinischWestfälische Technische Hochschule in Aachen, Germany, created a 3-dimensional model of the cardiovascular system for flow dynamic evaluation, based on computed tomography and magnetic resonance imaging data. These data were collected in our clinic from a voluntary proband (one of the investigators). By a division of this geometric figure into approximately 4.5 million small tetrahedron volumes, a calculation mesh was generated within the model. Walls were modeled by triangles, and flow equations were subsequently performed in each unit. The position of the cannula tip varied to estimate changes of flow in the VA.

A flow of 2.5 to $3.0 \mathrm{~L} / \mathrm{m}^{2} / \mathrm{min}$ through the arterial cannula was hypothesized for arterial inflow of regular extracorporeal circulation (in contrast to low flow settings, eg, selective antegrade cerebral perfusion). Blood was considered a non-Newtonian fluid. The stream was regarded as turbulent because of the pipe flow within the arterial cannula, and a fixed systemic pressure of $55 \mathrm{~mm} \mathrm{Hg}$ was assumed.

The blood flow in the cardiovascular system is laminar for regular flow conditions. However, the flow close to the cannula tip is turbulent, because the Reynolds number in this region increases far more than 2000. Under simulated conditions (approximate values), the Reynolds number can be calculated as follows: $>\operatorname{Re}=$ rho $\cdot \mathrm{v} \cdot \mathrm{d} / \mathrm{ny}=6306.7$

Density of the blood (rho) $=1056 \mathrm{~kg} / \mathrm{m}^{3}$

Maximum velocity $(\mathrm{v})=4.3 \mathrm{~ms}$

Diameter of the cannula $(\mathrm{d})=0.005 \mathrm{~m}$

Dynamic velocity of the blood (ny) $=0.0036 \mathrm{~Pa} / \mathrm{s}$.

Because the cannula tip is the main region of interest concerning this study, the shear stress transport turbulence model was chosen. Furthermore, the results in terms of total flow in the cardiovascular system do not vary with laminar or turbulent model assumption. This has a stronger effect on wall shear stress (WSS) due to energy loss of the fluid based on wrong turbulence assumptions in case of laminar flow or the effect of turbulent WSS for high/turbulent conditions. Because WSS was not part of the investigations of this study, the assumption of a shear stress transport turbulence model for the whole system is acceptable.

For analysis, the computer software ANSYS CFX 11.0 (Ansys Inc, Otterfing, Germany) was used. This software has been used for other cardiovascular research concerning hemodynamics and blood flow patterns. ${ }^{9-13}$ Supra-aortic flow was considered to be safe if there was no blockage of the VA's orifice or negative intravascular mass flow (Figure 1).

\section{RESULTS \\ Anatomic Study}

The average distance between the end of the distal segment of the right AA and the VA's origin was $8.2 \mathrm{~cm}$ $( \pm 0.69 \mathrm{~cm})$ in female subjects and $8.7 \mathrm{~cm}( \pm 0.80 \mathrm{~cm})$ in male subjects. The minimum distance was $7.2 \mathrm{~cm}$.

The mean distance from the end of the SA to the VA origin was $6.0 \mathrm{~cm}( \pm 0.50 \mathrm{~cm})$ in female subjects and $6.5 \mathrm{~cm}$ $( \pm 0.91 \mathrm{~cm})$ in male subjects. The minimum was $5.6 \mathrm{~cm}$ in female and male corpses. However, the observed differences between the measurements in female and male corpses were not statistically significant. The anatomic measurement data are summarized in Table 1.

\section{Computer Model for Hemodynamic Calculations}

Figure 2 shows a graphic illustration of our flow dynamic calculations depending on the proximity of the cannula tip to the orifice of the VA. Position 1 demonstrates that partial blocking of the right VA can lead to a negative intravascular mass flow and therefore to a steal phenomenon. Contrary to this, a more lateral position of the cannula (position 2) seems to lead to an improved supra-aortic perfusion. The mass flow (liters/minute) within the right VA depending on the distance between the cannula tip and the VA's origin is shown in Figure 3. Calculations for the right carotid artery point out a slight hyperperfusion compared with aortic cannulation.

\section{Discussion}

The present study was undertaken to specify safe cannulation sites with optimal cerebral perfusion after direct AA cannulation. We measured a mean distance of $8.5 \mathrm{~cm}$ from the AA and $6.7 \mathrm{~cm}$ from the SA to the origin of VA. Critical flow dynamics in the right supra-aortic vessels could be observed if the tip of the arterial cannula was in close position to the VA's origin or if it blocked the VA's orifice. Although selective cerebral perfusion through AA cannulation has proven advantages by reducing neurologic complications and mortality in aortic arch surgery, ${ }^{1-3}$ the technique of AA cannulation itself seems to have an impact on neurologic outcome. ${ }^{5-7}$ Many groups prefer to anastomose a side graft to the artery. We routinely use this technique if extracorporeal membrane oxygenation support is needed in patients with cardiac and respiratory failure. However, we and others observed a relative 


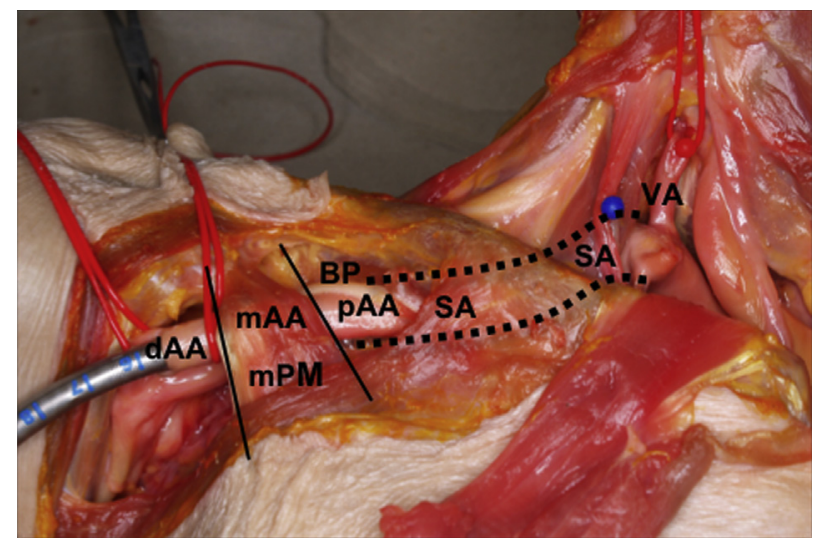

FIGURE 1. Anatomic preparation of an 80-year-old subject. Dissection and arterial cannulation of the distal segment of the right AA with its relation to the VA (red pin), brachial plexus, and minor pectoralis muscle (margins are marked by solid lines). The cannula is inserted into the distal AA. The dashed lines describe the course of the SA. Because the major pectoralis muscle is not divided from the clavicle, the distal segment of the SA is hidden. $B P$, Brachial plexus; $d A A$, distal segment of the right axillary artery; $m A A$, middle segment of the right axillary artery; $m P M$, minor pectoralis muscle; $p A A$, proximal segment of the right axillary artery; $S A$, subclavian artery; $V A$, vertebral artery.

hyperperfusion of the right arm in some cases, usually when the artery had a small caliber. This indicates that flow distribution to the body is uncertain once a double outflow is created, and thus effective body perfusion might be reduced.

This problem could be avoided by the direct cannulation technique, and at least for the duration of a complicated surgical procedure, there are no reports of serious ischemia of the cannulated arm. The collateral system in this area is strong, so critical malperfusion is not anticipated. However, arterial cannulation via a side graft might reduce the risk for local complications. ${ }^{6}$ We prefer direct cannulation of the AA. Cases of severe sclerosis prohibiting direct cannulation are rare. If direct insertion is not feasible, cannulation is attempted with the help of a guidewire and abandoned if the risk of trauma to the vessel is too high.

Our flow dynamic computer model provides new information regarding the perfusion characteristics of the right

TABLE 1. Distances of different arterial cannulation sites to the orifice of the right vertebral artery

\begin{tabular}{lrccccc}
\hline & $\mathbf{n}$ & Minimum & Maximum & Mean & $\mathbf{\pm}$ & $\mathbf{9 5} \%$ \\
\hline dAA (total) & 14 & 7.2 & 9.8 & 8.5 & & \\
dAA (male) & 7 & 7.6 & 9.8 & 8.7 & 0.80 & $7.92-9.40$ \\
dAA (female) & 7 & 7.2 & 9.0 & 8.2 & 0.69 & $7.61-8.88$ \\
SA (total) & 14 & 5.6 & 7.8 & 6.7 & & \\
SA (male) & 7 & 5.6 & 7.8 & 6.5 & 0.91 & $5.70-7.39$ \\
SA (female) & 7 & 5.6 & 7.0 & 6.0 & 0.50 & $5.57-6.49$ \\
\hline
\end{tabular}

Shown are the distances of different arterial cannulation sites to the origin of the right VA. Measurements were taken in 14 human corpses ( 7 male and 7 female), and results are presented in centimeters. $d A A$, End of the distal segment of the right axillary artery; $S A$, end of the distal segment of the right subclavian artery.
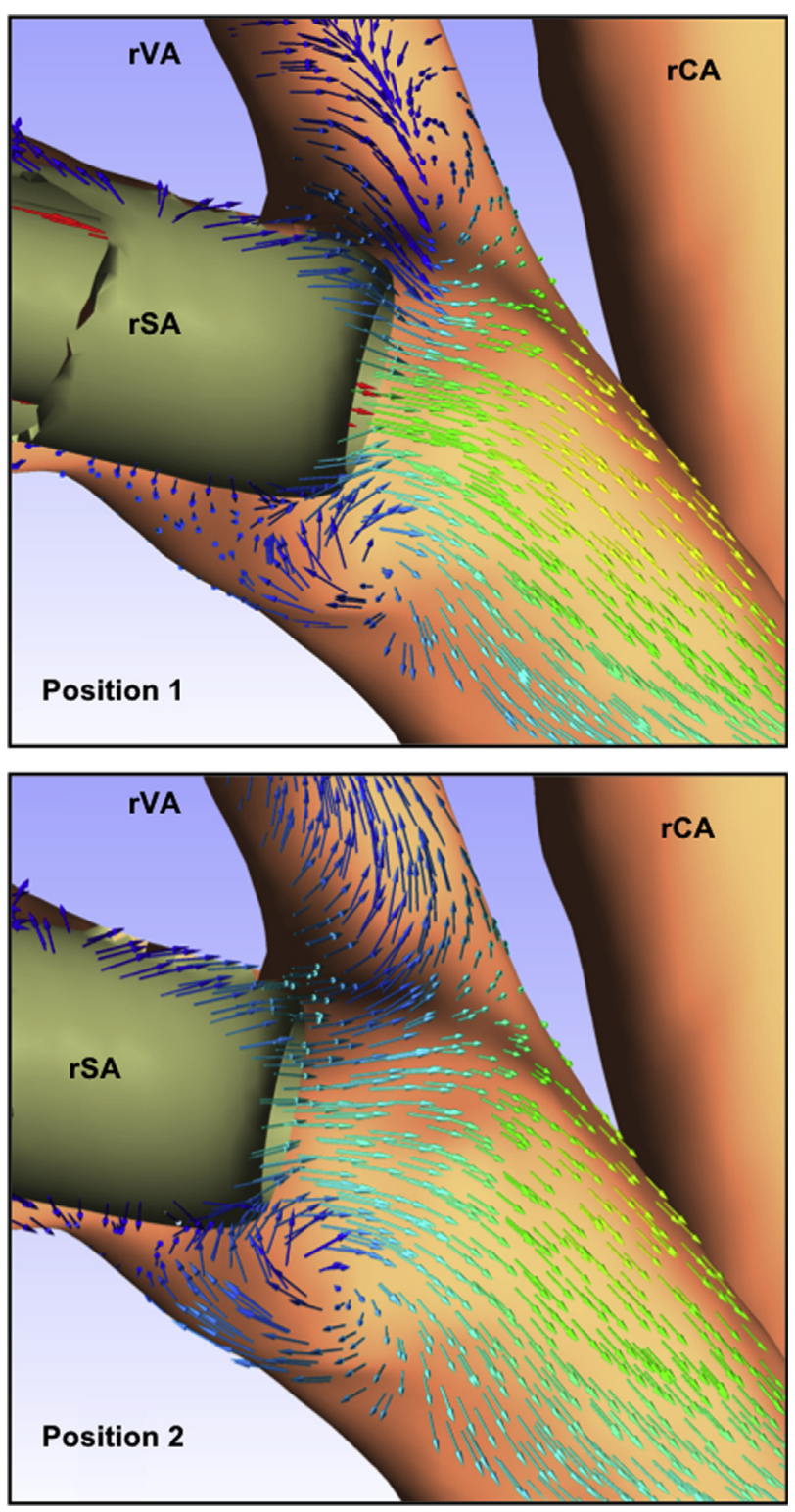

FIGURE 2. Changes of the intravascular flow dynamics within the right VA depending on 2 different positions (position $1=$ upper figure and $2=$ lower figure $)$ of the arterial cannula tip within the right SA, right VA, and right carotid artery. The color of the arrows shows the velocity. Position 1 demonstrates negative intravascular flow when the arterial cannula is positioned within the orifice of the right VA. $r C A$, Right carotid artery; $r S A$, right subclavian artery; $r V A$, right vertebral artery.

VA and SA. Because the right VA usually arises from the $\mathrm{SA},{ }^{14}$ cases of a VA origin in the bifurcation of the brachiocephalic trunk were not taken into consideration and simulated. Compared with direct aortic cannulation, a slight hyperperfusion of the right carotid artery can be observed during axillary inflow.

We simulated several perfusion scenarios with different positions of the cannula tip that might occur after SA cannulation. Position of the cannula tip at the orifice of the

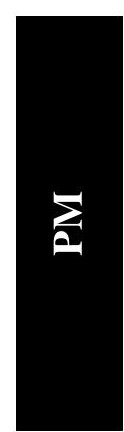




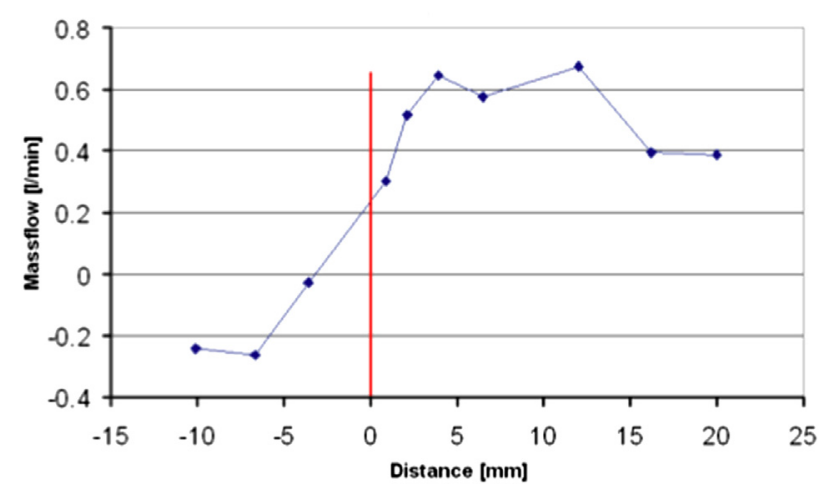

FIGURE 3. Intravascular mass flow (L/min) depending on different distances $(\mathrm{mm})$ of the arterial cannula tip. Negative distance values mean that the cannula's tip is already positioned beyond the VA's origin. The data show that a negative intravascular mass flow within the right VA can be observed when the cannula is directly positioned within the dexter VA origin or beyond that.

VA may cause a steal phenomenon by reversing blood flow. This model-based observation might lead to an imbalanced cerebral perfusion and therefore be a reason for the neurologic injuries observed in an in vivo ${ }^{15}$ or in vitro model. ${ }^{16}$ This potential danger might be aggravated in cases of close proximity of the right carotid and VA. In 2008, Shin and coworkers ${ }^{17}$ published a morphometric study that demonstrated a distance between the right common carotid artery and the right VA that ranged from 0.0 to $23.3 \mathrm{~mm}$. There are several other influencing factors, such as arterial diameter, cannula size, and flow through the cannula that have to be taken into account, but a detailed analysis of all these factors or a calculation of all anatomic variations with respect to their influence on vertebral flow was not the aim of this study. It rather provides information concerning the possibility of reversed vertebral flow for cannula positions too close to the VA in correspondence with clinical experience.

The relationship between cannula size and artery is of major importance. Under optimal conditions, the cannula should be nearly the size of the artery, as assumed in the numeric model. A larger cannula would reduce the jet effect but cannot be used because of physiologic constraints. A smaller cannula would cause an even stronger jet effect and thereby most likely increase the effect of suction from the VA. However, the numeric model provides the possibility to test novel cannula designs for their potential to neglect the suction effect and thereby increase patient outcome. This design development is currently ongoing.

An advantage of the SA cannulation in arch surgery is the possibility of continuous cerebral perfusion ${ }^{1,18}$ and even the avoidance of deep hypothermia. ${ }^{19}$ For surgery in patients at high risk for cardiac or aortic emboli, subclavian cannulation avoids embolization into the right brain and even limits emboli to the left hemisphere. ${ }^{20} \mathrm{~A}$ burst of atheroemboli can be detected by transcranial Doppler during direct aortic cannulation. ${ }^{21}$ For both of these reasons, we started to use axillary cannulation in patients with increased risk for cerebral complications. In more than 100 such patients, we were able to avoid major focal cerebral lesions.

The present investigation indicates that negative flow dynamics within the right VA might be avoided by moving the cannulation site laterally. Nevertheless, anatomic variation of the Willis circle has to be taken into account as an important influencing factor. ${ }^{22}$

Because the anatomic distance between the middle segment of the AA is more than $7 \mathrm{~cm}$ on average and at least $5.5 \mathrm{~cm}$, a save position can be achieved by cannulation of the AA at the minor pectoralis muscle and an insertion length of 3 to $4 \mathrm{~cm}$. The diameter of the artery still usually allows insertion of an $18 \mathrm{~F}$ cannula, keeping mean arterial line pressure at $324 \mathrm{~mm} \mathrm{Hg}$ even at pump flows at 3 $\mathrm{L} / \mathrm{min} / \mathrm{m}^{2}$. The lateral dissection is more direct and technically easier than the exposure of the distal SA segment. No muscles have to be transected, the course is more superficial, there is less danger to the pectoral nerve, and the point of encircling can be chosen medial or lateral to the minor pectoralis muscle, wherever it is more convenient. The distal segment is in close proximity to the fasciculi of the brachial plexus, but those can usually easily be dissected off bluntly. We initially observed 2 patients with palsy of the right hand in our series of more than 100 patients, but when the problem became overt, more careful dissection at these point avoided further complications of this kind. In our series of distal subclavian cannulation, we observed 1 symptomatic transection of the pectoral nerve. The surgical technique, such as using a side graft or not, performance of cannula insertion with or without guide wire, type of cannula, and position of the cannulation site, can have an impact on neurologic complications.

\section{Study Limitations}

For our numeric calculation, the following limitations must be considered: One important restriction of the described setup is the model of rigid walls, so elastic vascular tones cannot be considered. As a matter of principle, compliance was therefore always neglected, which can lead to imprecision. This shortcoming cannot be verified by experimental testing, because walls are inelastic too. Moreover, in vivo circumstances corresponding to our model-based terms have to be proven.

The anatomic preparation does not exactly resemble the surgical dissection, the amount of retraction of the artery after encircling with loops is especially difficult to measure. However, measurements of the anatomic distances are precise. The diameter of the vessel is based on the reconstruction, which might affect the absolute but not the relative flow values. Therefore, the results for the relative cannula position are still valid. 
Furthermore, the number of 14 examined corpses is too low to make definite conclusions. Further investigations should be undertaken to investigate the influence of the dissection site, insertion length of the cannula, and other factors on cerebral perfusion.

\section{CONCLUSIONS}

Our anatomic and flow dynamic evaluations indicate that an interference of the arterial cannula tip and the VA's origin might cause a subclavian steal phenomenon. Reversed intravascular blood flow can lead to cerebral malperfusion after direct SA or AA cannulation. A more protective arterial inflow could be achieved by a more lateral insertion, close to the middle segment of the AA. The avoidance of manipulation of a sclerotic aorta and a washout of debris by the reversed flow in the brachiocephalic trunk might be an additional beneficial factor.

\section{References}

1. Halkos ME, Kerendi F, Myung R, Kilgo P, Puskas JD, Chen EP. Selective antegrade cerebral perfusion via right axillary artery cannulation reduces morbidity and mortality after proximal aortic surgery. J Thorac Cardiovasc Surg. 2009; 138:1081-9.

2. Ogino H, Sasaki H, Minatoya K, Matsuda H, Tanaka H, Watanuki H, et al. Evolving arch surgery using integrated antegrade selective cerebral perfusion: impact of axillary artery perfusion. J Thorac Cardiovasc Surg. 2008;136:641-8.

3. Etz CD, Plestis KA, Kari FA, Silovitz D, Bodian CA, Spielvogel D, et al. Axillary cannulation significantly improves survival and neurologic outcome after atherosclerotic aneurysm repair of the aortic root and ascending aorta. Ann Thorac Surg. 2008;86:441-7.

4. Fleck T, Ehrlich M, Czerny M, Hutschala D, Tschernko E, Mares P, et al. Cannulation of the axillary artery: the decision between direct cannulation and cannulation via side graft. J Thorac Cardiovasc Surg. 2005;53:154-7.

5. Sabik JF, Nemeh H, Lytle BW, Blackstone EH, Gillinov AM, Rajeswaran J, et al. Cannulation of the axillary artery with a side graft reduces morbidity. Ann Thorac Surg. 2004;77:1315-20.

6. Yilik L, Emrecan B, Kestelli M, Ozsoyler I, Lafci B, Yakut N, et al. Direct versus side-graft cannulation of the right axillary artery. Tex Heart Inst J. 2006;33: $310-5$.
7. Svensson LG, Blackstone EH, Rajeswaran J, Sabik JF III, Lytle BW, GonzalesStawinski G, et al. Does the arterial cannulation site for circulatory arrest influence stroke risk? Ann Thorac Surg. 2004;78:1274-84.

8. Leitz KH. Zugangswege in der Gefäßchirurgie. Berlin: Springer-Verlag; 1981:26-8, 32-3.

9. Khalafvand SS, Ng EYK, Zhong L, Hung TK. Fluid-dynamics modeling of the human left ventricle with dynamic mesh for normal and myocardial infarction: preliminary study. Comput Biol Med. 2012;42:863-70.

10. Cheng Z, Tan FP, Riga CV, Bicknell CD, Hamady MS, Gibbs RG, et al. Analysis of flow patterns in a patient-specific aortic dissection model. J Biomech Eng. 2010;132:051007.

11. Bai-Nan X, Fu-Yu W, Lei L, Xiao-Jun Z, Hai Yue J. Hemodynamics model of fluid-solid interaction in internal carotid artery aneurysms. Neurosurg Rev. 2011;34:39-47.

12. Hose DR, Lawford PV, Narracott AJ, Penrose JM, Jones IP. Fluid-solid interaction: benchmarking of an external coupling of ANSYS with CFX for cardiovascular applications. J Med Eng Technol. 2003;27:23-31.

13. Berthier B, Bouzerar R, Legallais C. Blood flow patterns in an anatomically realistic coronary vessel: influence of three different reconstruction methods J Biomech. 2002;35:1347-56.

14. Yamaki K, Saga T, Hirata T, Sakaino M, Nohno M, Kobayashi S, et al. Anatomical study of the vertebral artery in Japanese adults. Anat Sci Int. 2006;81:100-6.

15. Gerdes A, Joubert-Huebner E, Esders K, Sievers H-H. Hydrodynamics of aortic arch vessels during perfusion through the right subclavian artery. Ann Thorac Surg. 2000;69:1425-30.

16. Joubert-Huebner E, Gerdes A, Sievers H- H. An in vitro evaluation of a new cannula tip design compared with two clinically established cannula-tip designs regarding aortic arch vessel perfusion characteristics. Perfusion. 2000;15:69-76.

17. Shin IY, Chung YG, Shin WH, Im SB, Hwang SC, Kim BT. A morphometric stud on cadaveric aortic arch and its major branches in 25 Korean adults: the perspective of endovascular surgery. J Korean Neurosurg Soc. 2008;44:78-83.

18. Zierer A, Moon MR, Melby SJ, Moazami N, Lawton JS, Kouchoukos NT, et al. Impact of perfusion strategy on neurologic recovery in acute type A aortic dissection. Ann Thorac Surg. 2007;83:2122-8.

19. Bakhtiary F, Dogan S, Dzemali O, Kleine P, Moritz A, Aybek T. Mild hypothermia (32 degrees $\mathrm{C}$ ) and antegrade cerebral perfusion in aortic arch operations. J Thorac Cardiovasc Surg. 2006;132:153-4.

20. Hedayati N, Sherwood JT, Schomisch SJ, Carino JL, Markowitz AH. Axillary artery cannulation for cardiopulmonary bypass reduces cerebral microemboli. J Thorac Cardiovasc Surg. 2004;128:386-90.

21. Mackensen GB, Ti LK, Phillips-Bute BG, Mathew JP, Newman MF, Grocott HP. Cerebral embolization during cardiac surgery: impact of aortic atheroma burden. Neurologic Outcome Research Group (NORG). Br J Anaesth. 2003;91:656-61.

22. Urbanski PP, Lenos A, Blume JC, Ziegler V, Griewing B, Schmitt R, et al. Does anatomical completeness of the circle of Willis correlate with sufficient crossperfusion during unilateral cerebral perfusion? Eur J Cardiothorac Surg. 2008; $33: 402-8$ 